\title{
Bayer Visual Acuity Changes Grade 3
}

National Cancer Institute

\section{Source}

National Cancer Institute. Bayer Visual Acuity Changes Grade 3. NCI Thesaurus. Code C128449.

Visual acuity loss greater than or equal to 6 lines. (ET DRS equivalent: each loss of 3 lines corresponds to halving the visual acuity) 